\title{
Modelo de un sistema de producción esbelto con redes de Petri para apoyar la toma de decisiones
}

\section{Lean production system model with Petri nets to support for decision making}

\author{
Alberto Morales Varela ${ }^{1} \quad$ Jorge Armando Rojas Ramírez ${ }^{1} \quad$ Luis Héctor Hernández Gómez ${ }^{1}$ \\ Ángel Morales González ${ }^{1}$ María Yanet Jiménez Reyes ${ }^{1}$ \\ Recibido 23 de abril de 2014, aceptado 25 de septiembre de 2014 \\ Received: April 23, $2014 \quad$ Accepted: September 25, 2014
}

\begin{abstract}
RESUMEN
En un mundo altamente dinámico, debido a los cambios tecnológicos, económicos, políticos y sociales en el entorno, se ha reconocido que los sistemas productivos desempeña un papel fundamental en la competitividad de una organización. En esta investigación se plantea la integración del pensamiento esbelto en la gestión de un sistema de producción mediante la técnica de simulación de eventos discretos empleando redes de Petri como metodología de apoyo en el proceso de toma de decisiones. La toma de decisiones en un sistema productivo esbelto es un proceso complejo, esto debido a sus características estáticas (estructurales), dinámicas (operacionales) y los desperdicios que se presentan. En el trabajo se diseñan un conjunto de estructuras que representan distintas fases en un proceso productivo de neumáticos y se demuestra que un modelo empleando la metodología de redes de Petri ayuda a tener una eficaz y eficiente toma de decisiones para la eliminación de desperdicios, esto gracias a las propiedades para modelar eventos discretos y analizar el comportamiento dinámico del sistema. Finalmente la fase de simulación permite analizar un conjunto de parámetros a través de un indicador sistémico OEE (Eficiencia General de los Equipos), y demostrar que la implementación en conjunto de métodos, técnicas y herramientas esbeltas genera sinergia en el sistema.
\end{abstract}

Palabras clave: Sistema de producción, producción esbelta, redes de Petri, simulación de eventos discretos, eficiencia general de los equipos.

\begin{abstract}
In a highly dynamic world, due to technological, economic, political and social changes in the environment, it has been recognized that the production systems play a critical role in the competitiveness of an organization. In this research the authors propose the integration of lean thinking in managing a production system using the technique of discrete event simulation using Petri nets as the support methodology in the process of decision making. Decision making in a lean production system is a complex process because it has static (structural) and dynamic (operational) characteristics and a set of waste presented in it. This paper presents the design of a set of structures that represent different stages in a production process of tires and that is it possible to prove that the design of a model, using the methodology of Petri nets, helps to have an effective and efficient decision making for waste disposal, this thanks to the properties of such nets to model discrete events and analyse the dynamic behaviour of the system. Finally the simulation allows analysing a set of parameters through a systemic indicator OEE (Overall Equipment Efficiency) and demonstrate that the implementation of methods, techniques and tools proposed in the lean production theory generate synergy in the system.
\end{abstract}

Keywords: Production system, lean production, Petri nets, discrete event simulation, overall equipment effectiveness.

1 Departamento de Ingeniería en Sistemas. ESIME Unidad Zacatenco. Instituto Politécnico Nacional. México, D.F.

E-mail: am_varela@prodigy.net.mx; jrojasr@ipn.mx; luishector56@hotmail.com; anmorales@ipn.mx;

maria.jimenez.reyes@gmail.com 


\section{INTRODUCCIÓN}

La producción esbelta es una teoría fundamental para el mejoramiento de la productividad y la competitividad de las empresas de manufactura y servicios. La globalización de mercados exige a las organizaciones mantener estándares de clase mundial en sus procesos, entendiendo que toda ventaja competitiva es temporal y existe la necesidad de someterse a constantes procesos de adaptación por parte de todos los miembros de la red de valor.

La adopción de paradigmas de producción, aplicación de metodologías, técnicas y herramientas a través del tiempo han mostrado tener una tendencia hacia el reduccionismo y se han enfocado a resolver situaciones específicas, aunado a lo anterior han dejado de considerar en determinados momentos el factor humano, considerado como uno de los desperdicios descritos en la teoría de producción esbelta.

Estableciendo que los sistemas de producción no solo responden a las restricciones cuantitativas bien definidas, sino también a las variaciones cualitativas que el factor humano introduce en su operación, y que la identificación y eliminación de desperdicios (entendido como desperdicio toda aquella actividad que no agrega valor, pero sí un costo y trabajo) requieren la implementación de un proceso continuo y sistemático a través de un modelo. Se identifica que uno de los problemas fundamentales al que se enfrentan las organizaciones es la necesidad de diseñar procesos con base en una visión sistémica que les permita identificar características emergentes y mejorar toda área de oportunidad en el sistema, a través de mejoras tecnológicas, económicas, sociales y políticas en sus procesos de gestión y producción.

La aportación de esta investigación es ofrecer una solución a la problemática mencionada, mediante la propuesta de un modelo sistémico a los responsables de la gestión de sistemas productivos, que caracterice un conjunto de estructuras a través de redes de Petri, el cual puede ser empleado como una herramienta que apoya en el proceso de estudio, análisis y toma de decisiones, para la mejora estratégica del sistema, empleando métodos, técnicas y herramientas eficientes propuestas en la teoría de producción esbelta.
El modelo permite identificar los diferentes tipos de relación que pueden establecerse entre los elementos y la sensibilidad que las variables tienen mediante la interacción y evolución a través del tiempo. Lo antes mencionado se obtiene en función del conjunto de decisiones estratégicas a través de las cuales se va a configurar la estructura productiva de la organización, estas incluyen el conjunto de decisiones tendentes a la mejora continua, mediante la elección, configuración y distribución de los factores productivos fijos y variables, instalaciones, sistemas de información, recurso humano y materiales, para que la capacidad de creación de valor, como medida de eficiencia del sistema productivo, sea la máxima posible.

El trabajo parte definiendo conceptualmente la relación que existe entre la ciencia de sistemas y la cadena de suministro, posteriormente el diseño del modelo se ubica en el contexto del eslabón de manufactura.

\section{MARCO CONCEPTUAL}

\section{Análisis sistémico de la cadena de suministros}

En su acepción más amplia, la teoría general de sistemas se presenta como una forma sistemática y científica de aproximar y representar la realidad [1]. Al mismo tiempo, juega un papel muy importante en la investigación para orientar los análisis desde una perspectiva holística e integradora [3].

Hasta la aparición de la teoría de sistemas, los diferentes planteamientos formulados para el estudio de las organizaciones se realizaban desde una concepción limitada al entenderla como un sistema cerrado. Sin embargo, una concepción integral de dicho planteamiento rompió con este paradigma tradicional introduciendo el concepto de sistema abierto [4-5]. Desde esta perspectiva el estudio de las organizaciones supone una necesaria interrelación entre el sistema estudiado y su entorno, de tal manera que se asume la existencia de una serie de relaciones de interinfluencia constante e intercambio continuo de elementos [6-7].

Desde el punto de vista sistémico, una cadena de suministro se define como un sistema integrado por diversas empresas relacionadas entre sí, que trabajan en armonía con el propósito de alcanzar objetivos comunes tanto de la organización como 
de sus integrantes, que mantienen una constante dinámica de interacción con su medio ambiente [9].

En la Figura 1 se muestran los elementos principales que conforman una cadena de suministro, se encuentran por una parte los procesos de transformación, abastecimiento y almacenamiento tanto de insumos como de productos terminados, y por otra, los de distribución, colocación y entrega final del producto [9-10].

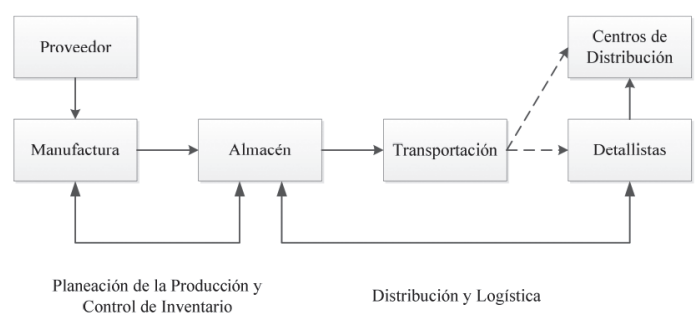

Figura 1. Proceso de cadena de suministro.

\section{Producción esbelta}

El paradigma de producción esbelta está basado en su totalidad en el Sistema de Producción de Toyota (TPS), nació en Japón y fue concebido por los grandes gurús, entre ellos: Sakichi Toyoda, Kiichiro Toyoda, Eijy Toyoda, Taiichi Ohno, Shigeo Shingo y W. Edwards Deming.

En 1990 James P. Womack, Daniel T. Jones y Daniel Roos, del Massachusetts Institute of Technology (MIT) analizaron la evolución de los sistemas de gestión de producción, y a través de esto definen un conjunto de principios y conceptualizan el paradigma de producción esbelta.

Producción esbelta es un paradigma que tiene como base un proceso continuo y sistemático de identificación y eliminación de desperdicios [11]. Establece una filosofía de gestión enfocada a la creación de valor, para poder entregar el máximo valor para los clientes, utilizando para ello los mínimos recursos necesarios. Esta eliminación sistemática se lleva a cabo mediante trabajo con equipos de personas bien organizados y capacitados [12].

La creación de valor se focaliza en la reducción de los ocho tipos de desperdicios en productos manufacturados eliminando el desperdicio, mejorando la calidad y reduciendo el tiempo de producción y el costo [13].
Se han identificado ocho tipos de desperdicios que no agregan valor al proceso de manufactura, estos desperdicios son: sobreproducción, inventario, reparación y rechazos, movimientos innecesarios, procesamiento incorrecto, tiempo de espera y talento humano [14-15]. En la Tabla 1 se clasifican los métodos, herramientas y técnicas que se proponen en la teoría de producción esbelta, con el objetivo de eliminar los desperdicios.

Tabla 1. Metodologías, herramientas y técnicas en Producción Esbelta.

\begin{tabular}{|c|c|}
\hline \multirow{4}{*}{ Herramientas básicas } & Kaizen \\
\hline & $\begin{array}{l}\text { VSM (Value Stream } \\
\text { Mapping) }\end{array}$ \\
\hline & $5 \mathrm{~S}^{\prime} \mathrm{s}$ \\
\hline & Andon \\
\hline \multirow{3}{*}{$\begin{array}{r}\text { Herramientas para } \\
\text { mejorar la efectividad }\end{array}$} & $\begin{array}{l}\text { TPM (Total Productive } \\
\text { Maintenance) }\end{array}$ \\
\hline & Manufactura Celular \\
\hline & SMED \\
\hline \multirow{6}{*}{$\begin{array}{l}\text { Herramientas para } \\
\text { mejorar la calidad }\end{array}$} & $\begin{array}{r}\text { Herramientas de Calidad } \\
\text { (Herramientas Estadísticas) }\end{array}$ \\
\hline & Soifuku \\
\hline & Poka Yoke \\
\hline & Jidoka \\
\hline & 8 D's \\
\hline & Six Sigma \\
\hline \multirow{4}{*}{$\begin{array}{l}\text { Herramientas para el } \\
\text { control de materiales y } \\
\text { producción }\end{array}$} & Kanban \\
\hline & Heijunka \\
\hline & Takt Time \\
\hline & Shojinka \\
\hline
\end{tabular}

\section{METODOLOGÍA}

Un proyecto de simulación es de naturaleza dinámica, para llevarla a cabo se proponen las siguientes fases, estas son descritas en [24]:

1. Formulación del problema.

2. Diseño del modelo conceptual.

3. Recolección de datos.

4. Construcción del modelo.

5. Validación del modelo.

\section{Redes de Petri}

El modelo se desarrolló empleando la metodología de redes de Petri (RdP). Una RdP es un grafo orientado en el que intervienen dos clases de nodos, llamados lugares y transiciones, unidos por arcos orientados, de manera alternada. Un lugar puede tener un número positivo o nulo de marcas [16]. 
Las RdP proporcionan una herramienta matemática y gráfica de modelado para la descripción formal de sistemas de eventos discreto, tienen un alto potencial descriptivo y permiten una representación clara de sistemas cuya dinámica se caracteriza por la concurrencia, sincronización, exclusión mutua y conflictos, facilitando con ello la descripción y la elaboración de modelos, así como la posterior implantación de sistemas [24]. Como herramienta gráfica, las RdP son empleadas como una representación de los sistemas, utilizando marcas para simular la dinámica y las operaciones concurrentes; como herramienta matemática, las RdP permiten obtener modelos gobernados por el comportamiento del sistema [17].

\section{Definición formal}

Una red de Petri generalizada es una cuádrupla $\mathrm{R}=<\mathrm{P}, \mathrm{T}, \alpha, \beta>$ tal que:

$\mathrm{P}$ es un conjunto finito y no vacío de lugares.

$\mathrm{T}$ es un conjunto finito y no vacío de transiciones. $\mathrm{P} \cap \mathrm{T}=\Phi$; es decir, lugares y transiciones son conjuntos disjuntos.

$\alpha: \operatorname{P} \times T \in \mathbf{N}$ es la función de incidencia previa

$\beta: \mathrm{T} \times \mathrm{Pe} \mathbf{N}$ es la función de incidencia posterior.

\section{Definición gráfica}

Una RdP se representa esquemáticamente por lugares y transiciones en una gráfica orientada. Tal como se observa en la Figura 2, los lugares se representan por circunferencias y las transiciones por barras. Existe un arco que va del lugar $\mathrm{p}_{\mathrm{i}}$ a la transición $\mathrm{t}_{\mathrm{j}}$ si y sólo si $\alpha\left(\mathrm{p}_{\mathrm{i}}, \mathrm{t}_{\mathrm{j}}\right) \neq 0$. Análogamente, existe un arco que va de la transición $\mathrm{t}_{\mathrm{k}}$ al lugar $\mathrm{p}_{\mathrm{i}}$ si y sólo si $\beta\left(\mathrm{t}_{\mathrm{k}}, \mathrm{pi}\right) \neq 0$. Cada arco se etiqueta con un entero natural, $\alpha(p, t)$ o $\beta(t, p)$, que se denomina ponderación del arco. Por convención, un arco no etiquetado posee una ponderación unitaria [18].

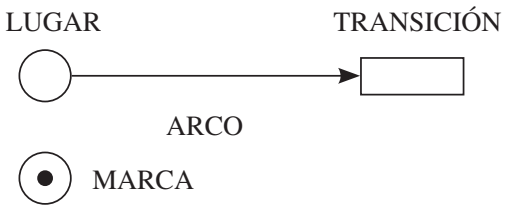

Figura 2. Identificación de componentes en una red de Petri.

\section{MODELO DEL SISTEMA}

El diseño del modelo consiste en la propuesta de un conjunto de estructuras que caracterizan los desperdicios identificados a través de la teoría de producción esbelta, los cuales se presentan en diferentes etapas de un sistema productivo. El modelo tiene como objetivo identificar y representar componentes, flujos de información y flujos de materiales a través del formalismo de RdP, y eliminar aquellos desperdicios mediante la reconfiguración de la red.

La reconfiguración está en función de un conjunto de indicadores de desempeño. Estos permiten evaluar el desempeño de la red de forma global y permiten tener una visión para decidir si la mejora establecida es una propuesta viable.

\section{Descripción del sistema de producción de neumáticos}

El sistema en estudio es una planta de producción de neumáticos localizada en Ciudad de México. El proceso debe garantizar que cada neumático reúna los más altos estándares de calidad, motivo por el que la presente investigación integra el paradigma de producción esbelta al proceso. De forma breve, el proceso se describe a través de las siguientes etapas:

1. El caucho natural y sintético es cortado en piezas pequeñas y sometido a calentamiento, con la finalidad de simplificar y acelerar el proceso de mezcla.

2. Esta mezcla, que consiste en el recorte longitudinal, molienda, es realizada después de añadir varios aditivos como azufre, óxido de zinc y carbón negro.

3. En este punto el caucho puede ser procesado en una de las cinco formas diferentes de producción para manufacturar uno de los tres tipos de productos que forman parte del neumático.

Estos 3 elementos son:

a) Alerón. Los alerones son producidos colocando el caucho mezclado en un estrujador para formar tiras de caucho que luego son cortados a las longitudes deseadas, y endurecidos en una prensa de curado para formar los alerones.

b) Cámaras. Ya que las propiedades físicas del caucho usado para producir las cámaras difieren del caucho usado para producir los neumáticos 
y los alerones, se requerirá de un proceso adicional. El caucho mezclado es pasado a través de un colador antes que sea colocado en la mezcladora de rodillos. Después de mezclado, un estrujador forma las tiras de caucho que son unidas y prensadas para formar la cámara del neumático.

\section{c) Neumáticos.}

La línea de manufactura de neumáticos contempla las siguientes fases:

i. Huella o banda de rodadura: El caucho mezclado es alimentado en un estrujador para formar la huella o banda del neumático. Después de este proceso, la huella o banda es enviada a la máquina formadora de neumáticos.

ii. Capas: El material usado como la capa de los neumáticos es una tela engomada hecha del caucho y cordones de nylon en una calandria de rodillos múltiples. Después que cada capa de este material es cortada en un ángulo específico, es enviado a la máquina formadora de neumáticos.

iii. Listones: Los listones en forma de anillo usados para formar los listones de las llantas son hechos automáticamente por una serie de máquinas que usan alambres recubiertos de caucho para formar lazos que son envueltos con la tela de nylon de caucho producida en el paso anterior. Luego los listones son enviados a la máquina formadora de neumáticos.

iv. Ensamble: La tela de nylon engomada, los listones, y la huella o banda son colocados, en ese orden, en la máquina formadora de neumáticos. Las capas de la tela de nylon engomada son envueltas con los listones en dirección opuesta para formar el centro del neumático. La banda de rodamiento es colocada alrededor del perímetro externo del centro del neumático y doblado conjuntamente formando el neumático verde.

v. Curado: El neumático verde es colocado en una prensa de curado que suministra calor, vapor y presión para formar el neumático vulcanizado.

vi. Acabado: Los neumáticos son pulidos, recortados, balanceados, inspeccionados y envueltos, completando de esta manera el proceso.

Una vez definida la lógica de operación del sistema, se desarrolla un modelo empleando la metodología de RdP. Tal como se muestra en la Figura 3, se representa el sistema de producción de una planta de neumáticos, se emplean transiciones determinísticas, estocásticas y temporizadas que son identificadas en la Tabla 4 del Anexo 1. Este modelo se basa en eventos discretos, los cuales tienen como objetivo identificar a sistemas en donde los eventos que cambian el estado del mismo ocurren en instantes espaciados en el tiempo [19].

En este trabajo se presenta el diseño de tres redes que representan el comportamiento de un sistema TPM (Total Productive Maintenance), un sistema SMED (Single Minute Exchange of Die) y un sistema Kanban. Las tres redes son integradas en la línea de producción de cámaras en las fases de tamizado, laminado, estrujado, revestido, montaje y prensado. Estas fases son identificadas en la red principal de la Figura 3, a través de los lugares p19, p20, p21, p22, p23 у p24.

\section{Red de Petri de un sistema TPM}

Uno de los sistemas importantes en que se basa el Kaizen es el TPM. El objetivo del mantenimiento de máquinas y equipos es conseguir un determinado nivel de disponibilidad de producción en condiciones de calidad exigible, a mínimo costo y con la máxima seguridad para el personal que las utiliza [20].

La estructura que se propone representa una operación de manufactura y el comportamiento del sistema cuando ocurre una falla en cualquiera de las máquinas o equipos. Este comportamiento se representa incorporando de manera repetida la estructura que se muestra en la Figura 4 en diferentes etapas dentro del sistema de producción.

Con el objetivo de mostrar la relación que existe entre el sistema y el modelo; observar cómo el disparo de una transición puede representar una operación del sistema; cómo la ejecución de la operación cambia el marcado; y cómo éste refleja el nuevo estado del sistema, se muestran las fases de construcción de la estructura básica de sistema TPM.

La RdP de la Figura 5 corresponde a la ejecución de una operación. Existe una transición al inicio, t1, en espera de ser habilitada externamente, con la generación de una señal para iniciar la operación. 


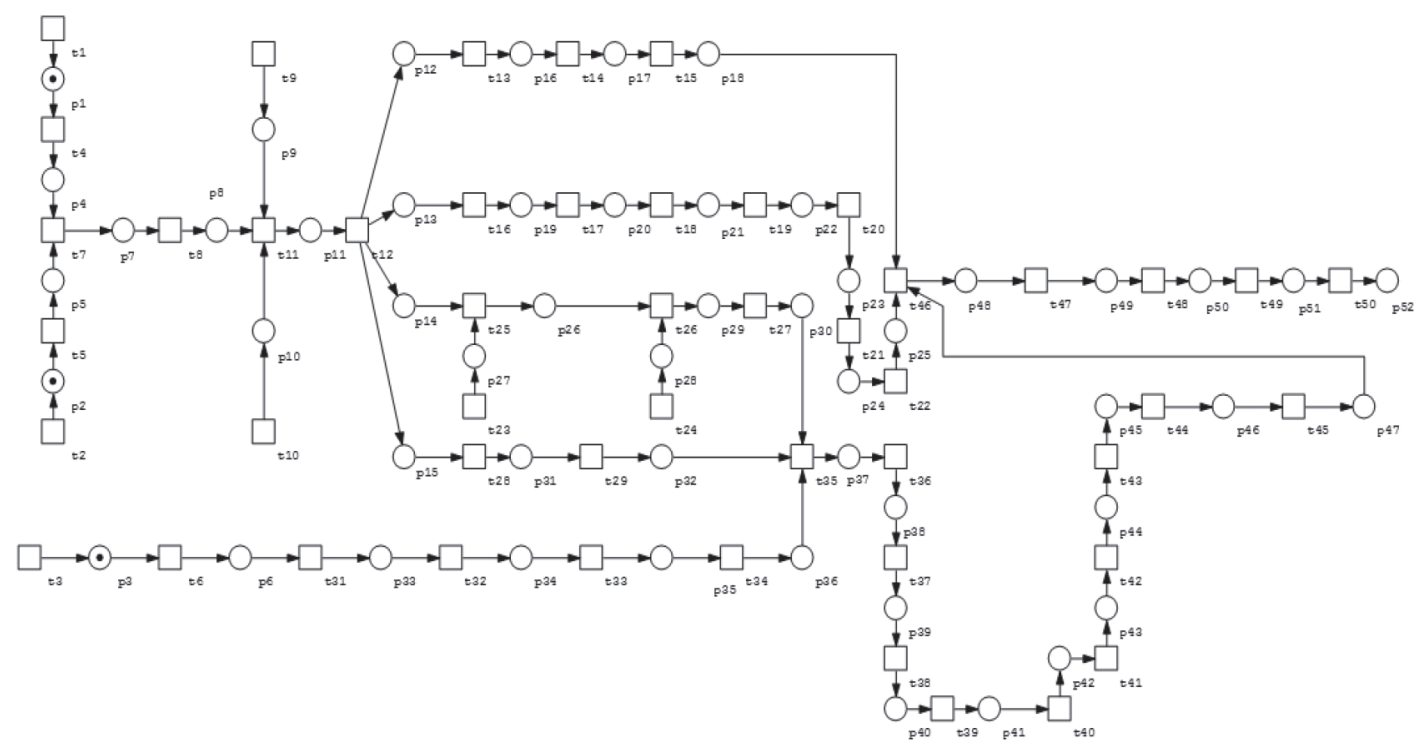

Figura 3. Red de Petri de una planta de producción de neumáticos.

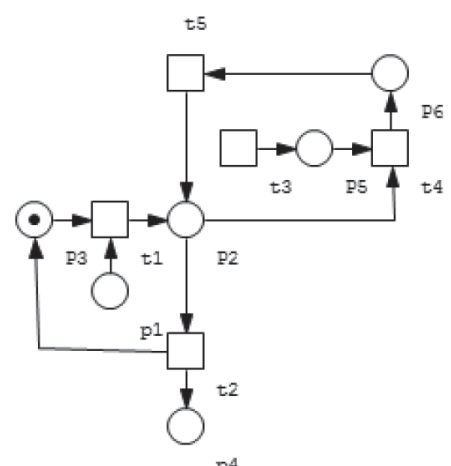

Figura 4. Modelo TPM mediante el uso de redes de Petri.

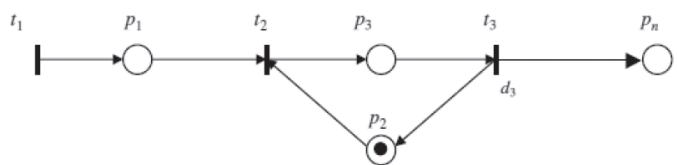

Figura 5. RdP de operación elemental en espera.

Después del disparo de $\mathrm{t} 1$, el marcaje de la red cambia, como se indica en la Figura 6, al ser ocupado por una marca el lugar p1.

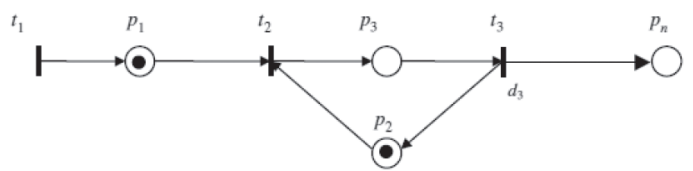

Figura 6. RdP de operación elemental al iniciar.
Es posible entonces que la red evolucione hacia la activación de p3 (una marca en este lugar), puesto que t2 está habilitada, ya que también hay una marca en p2. Ésta señala la disponibilidad de la máquina para la operación. Entonces, de acuerdo con la regla de disparo, cambia el marcaje de la RdP: las marcas de $\mathrm{p} 1$ y $\mathrm{p} 2$ desaparecen, para aparecer una en p3, como se observa en la Figura 7. La ausencia de marca en $\mathrm{p} 2$ indica que ahora la máquina ya no está libre para nuevas solicitudes de operación.

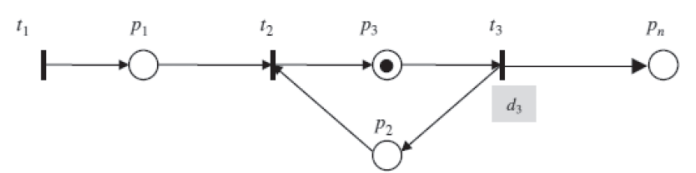

Figura 7. RdP de operación elemental.

El fin de la operación está regido por la temporización (d3) de la transición t3. Terminada su duración, desaparece la marca de p3, vuelve a aparecer una marca en $\mathrm{p} 2$ (indicando la disponibilidad del recurso) y se genera una marca en el lugar de salida pn, como se muestra en la Figura 8. El flujo puede volver a iniciarse en t1, para la ejecución de un nuevo ciclo.

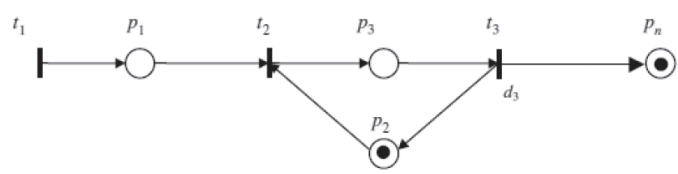

Figura 8. RdP de operación elemental al finalizar. 
Para la siguiente fase del ejemplo, se muestra el comportamiento del sistema cuando una falla se presenta en el proceso. Las RdP permiten representar en el sistema la aparición de fallas y la acción de reparación, como se indica a continuación. Se representa el sistema susceptible a fallas de la máquina, mediante la RdP de la Figura 9, en la que el lugar p5 activo indica que la falla se manifiesta, la transición 15 indica la ejecución de la reparación, y el lugar p6 el estado de reparación.

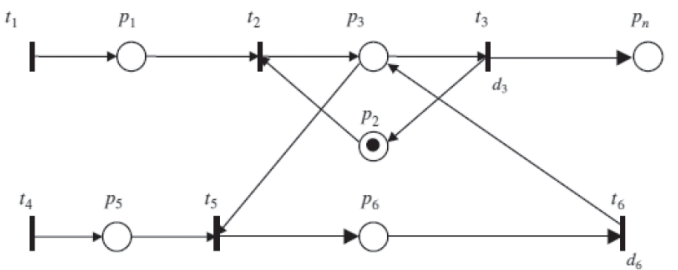

Figura 9. RdP de la operación con falla.

La transición $\mathrm{t} 4$ genera, en un instante determinado, la aparición de la falla. El lugar p5 se activa con una marca, de modo que en el momento de iniciarse la ejecución de la operación en p3, se dispara la transición t5. El marcaje de la red cambia entonces, únicamente con una marca en p6, como se ilustra en la Figura 10, se representa que la reparación se está llevando a cabo, con una duración de d6. Pasado este tiempo, la transición t6 se dispara y se restablece la fase de operación, en p3, para finalizar el ciclo de manera normal, entregando un producto terminado en el lugar pn.

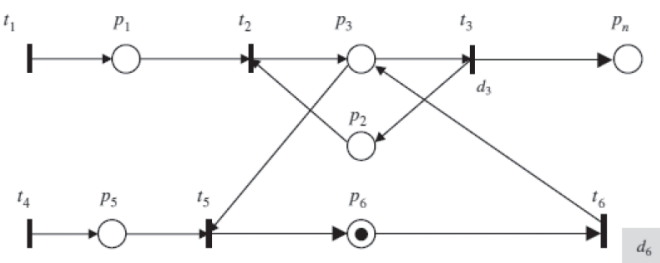

Figura 10. RdP de la operación de la reparación.

\section{Red de Petri de un sistema SMED}

SMED es un concepto el cual introduce la idea de que en general cualquier cambio de máquina o inicialización de proceso debería durar no más de 10 minutos, de ahí la frase single minute. Se entiende por cambio de herramientas el tiempo transcurrido desde la fabricación de la última pieza válida de una serie hasta la obtención de la primera pieza correcta de la serie siguiente.
Tabla 2. Lugares y Transiciones de la Red TPM.

\begin{tabular}{|c|l|}
\hline Elemento & \multicolumn{1}{|c|}{ Descripción } \\
\hline $\mathrm{t} 1$ & Inicio del programa de producción \\
\hline $\mathrm{t} 2$ & Inicio de la operación normal \\
\hline $\mathrm{t} 3$ & Término de la operación \\
\hline $\mathrm{t} 4$ & Aparición de una falla \\
\hline $\mathrm{t} 5$ & Suspensión de la operación \\
\hline $\mathrm{t} 6$ & Término de la reparación de máquina \\
\hline $\mathrm{p} 1$ & Producción en espera \\
\hline $\mathrm{p} 2$ & Máquina disponible \\
\hline $\mathrm{p} 3$ & Operación en ejecución \\
\hline $\mathrm{pn}$ & Salida de producción normal \\
\hline $\mathrm{p} 5$ & Máquina en falla \\
\hline $\mathrm{p} 6$ & Máquina en reparación \\
\hline
\end{tabular}

La estructura que se propone se muestra en la Figura 11, esta representa el proceso de cambio de un producto en una fase de la línea de manufactura dentro del sistema; cuando se requiere llevar a cabo el cambio, la transición t11 se sensibiliza, para que así la máquina en operación realice el paro y se lleve a cabo la reconfiguración del sistema, el lugar p5 indica que el proceso de cambios se está llevando a cabo, finalmente la transición t3 se sensibiliza y el marcaje evoluciona al lugar p6, el cual representa e indica que la reconfiguración ha sido llevada a cabo con éxito, de esa forma el sistema comienza la producción de un nuevo producto.

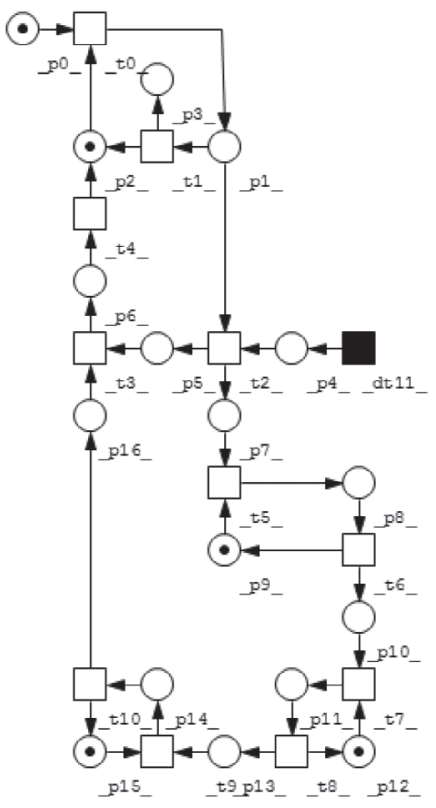

Figura 11. Modelo SMED mediante el uso de redes de Petri. 


\section{Red de Petri de un sistema Kanban}

El sistema pull es un sistema de comunicación que permite controlar la producción, sincronizar los procesos de manufactura con los requerimientos del cliente y apoyar fuertemente la programación de la producción [21]. En la Figura 12 se propone una estructura que representa un sistema kanban. Se proponen dos tipos, un kanban de retiro identificado por el lugar p22 y un kanban de producción identificado por los lugares p19, p20 y p21 [22].

La Figura 12 muestra tres estaciones de trabajo, están conformadas por una operación de maquinado, un depósito de piezas para cada estación y las etapas de flujo de materiales, de manera breve se describe el proceso; cuando existe demanda, se envía una orden de kanban de retiro al almacén, al mismo tiempo se envía una orden de kanban de producción para dar inicio a los procesos de manufactura, en caso de no existir producto en inventario, se envía una orden directamente para comenzar producción al inicio del proceso. La demanda es representada mediante una transición fuente, encargada de generar la demanda de forma aleatoria.

\section{SIMULACIÓN Y RESULTADOS}

El éxito de los paradigmas de producción modernos, tales como producción esbelta, depende en gran medida de la medición precisa y el análisis de los datos de producción.

La validación de la RdP está en función de un indicador de desempeño global, capaz de monitorear y mejorar la efectividad de un proceso productivo. Este indicador es el OEE (Overall Equipment Effectiveness).

El OEE es una herramienta de medición de eficiencia en una línea de producción o un equipo, se expresa como un porcentaje del producto de tres parámetros fundamentales de la producción: la disponibilidad, la eficiencia y la calidad, tal como se indica en la ecuación (1).

$\mathrm{OEE}=($ Disponibilidad $) \times($ Rendimiento $) \mathrm{x}$ (Calidad)

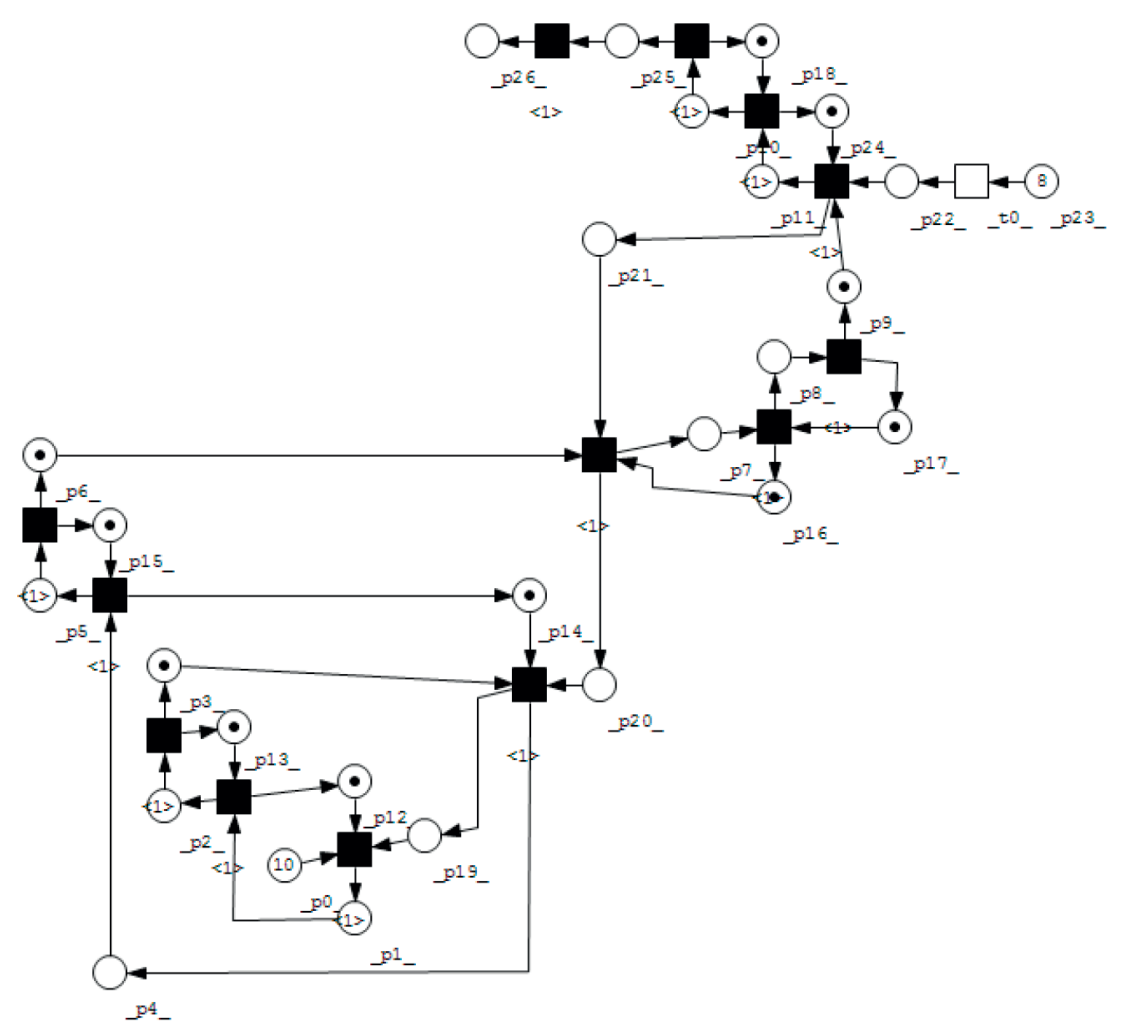

Figura 12. Modelo Kanban mediante el uso de redes de Petri. 
La Disponibilidad es la medida como el cociente entre el Tiempo Productivo y el Tiempo Disponible, para un periodo de producción determinado.

El Rendimiento es medido como el cociente entre la Producción Real y la Capacidad Productiva, para un periodo de producción determinado.

La Calidad es medida como el cociente entre la Producción Buena y la Producción Real.

La investigación busca integrar las estructuras desarrolladas a lo largo de todo el sistema productivo. Con el objetivo de ejemplificar cómo se desarrolla el proceso de validación de la red; las tres RdP esbeltas presentadas son integradas en la línea de producción de cámaras. El análisis se lleva a cabo través de un proceso de simulación mediante el uso del software Snoopy, desarrollado por investigadores de la Universidad de Brandeburgo [23]. Las redes expuestas contienen transiciones estocásticas con el objetivo de simular posibles eventos, como son: fallas de equipo y de piezas defectuosas. Por otro lado contienen transiciones temporizadas con el objetivo de simular el tiempo en que los equipos se mantienen sin operar.

A continuación se presenta el cálculo del desempeño de la red haciendo uso del indicador OEE, en el proceso de validación se considera un turno de trabajo de 24 horas, con una capacidad productiva de 2.500 piezas. Las gráficas presentadas se obtienen a través del análisis estocástico de la red de Petri, haciendo uso del módulo redes de Petri híbridas del software Snoopy.

\section{Disponibilidad en la línea de producción}

La disponibilidad se analiza a través de la gráfica de la Figura 13, mediante los lugares d1, d2, d3, $\mathrm{d} 4$, d5 y d6, en esta se muestran las paradas que existieron en el proceso, estas debido a factores como: tiempo de arranque y fallas. Los datos que se muestran en la Tabla 3, se obtienen a través del análisis de un conjunto de transiciones temporizadas haciendo uso del software.

Siendo el tiempo productivo en el proceso de 1.410 minutos y el tiempo total de 1.440 minutos, se aplica la ecuación que corresponde al indicador de disponibilidad, a través de esto, se obtiene como resultado un $97,9 \%$ de disponibilidad en el equipo.

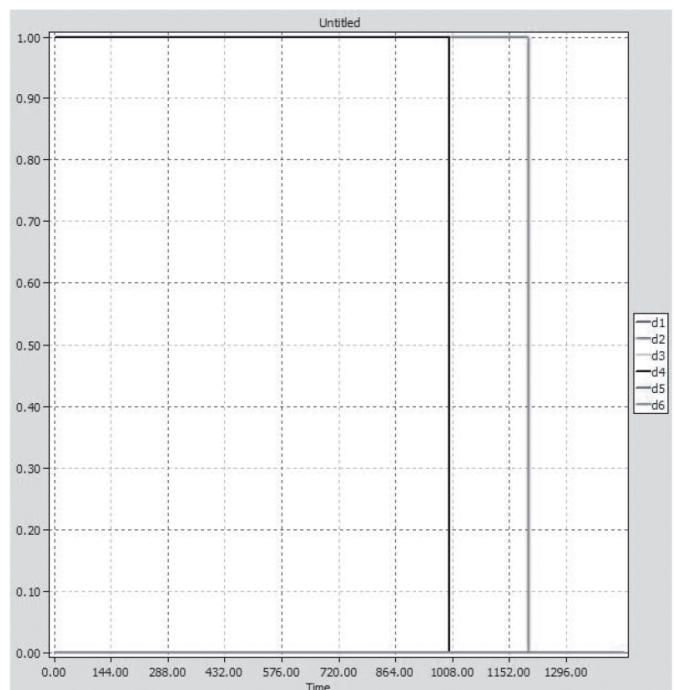

Figura 13. Disponibilidad en el proceso.

Disponibilidad $=($ Tiempo productivo real $)$ / (Tiempo disponible)

Disponibilidad $=1.410 / 1 \cdot 440=0,979$

Tabla 3. Disponibilidad en máquinas.

\begin{tabular}{|c|c|}
\hline Elemento Analizado & $\begin{array}{c}\text { Tiempo no activo } \\
\text { (minutos) }\end{array}$ \\
\hline Máquina 1 (d1) & 0 \\
\hline Máquina 2 (d2) & 20 \\
\hline Máquina 3 (d3) & 0 \\
\hline Máquina 4 (d4) & 10 \\
\hline Máquina 5 (d5) & 0 \\
\hline Máquina 6 (d6) & 0 \\
\hline
\end{tabular}

\section{Rendimiento en la línea de producción}

Posteriormente se analiza el rendimiento, para lo que es importante conocer el ritmo de producción del proceso a través del takt time, tiempo para satisfacer la demanda del cliente, desarrollado mediante la aplicación de la ecuación (3).

Tak time $=($ Tiempo total disponible por día $)$ / (Demanda diaria)

Tak time $=(1440 \mathrm{~min}) /(2500$ piezas $)=$ $0,576 \mathrm{~min} /$ pieza

Conocido el valor takt time y la capacidad de producción que corresponde a 2.500 piezas en 24 horas, la media de producción resulta de 104 


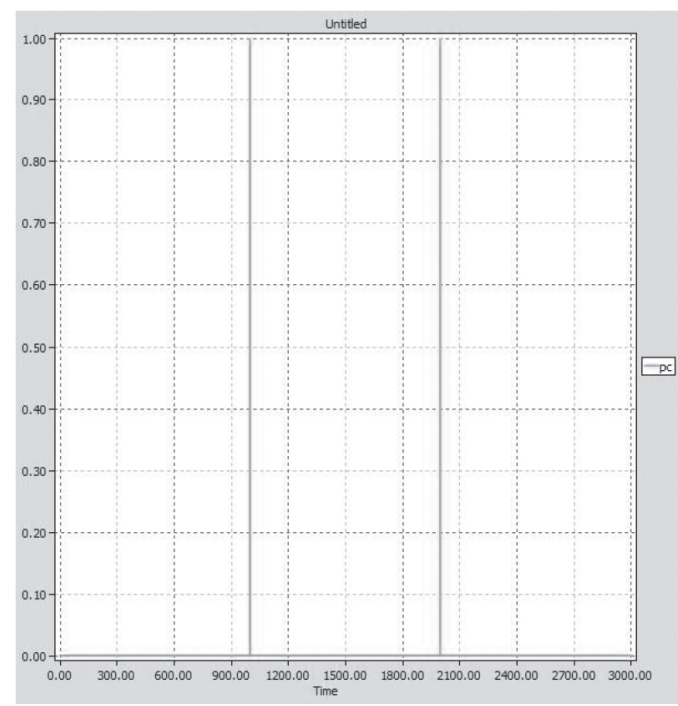

Figura 14. Calidad en el proceso.

piezas/hora. Teniendo el registro de 30 minutos de no disponibilidad durante el proceso, tenemos una producción de real de 2.447 piezas. Aplicando la ecuación (4), se obtiene que el rendimiento corresponde a un $97,8 \%$.

$$
\begin{gathered}
\text { Rendimiento }=(\text { Producción real }) / \\
(\text { Capacidad productiva })
\end{gathered}
$$

Rendimiento $=2.447 / 2.500=0,978$

\section{Calidad en la línea de producción}

El siguiente paso es conocer la calidad en el proceso, está la analizamos a través de la gráfica en la Figura 14, mediante el lugar pc, en ellas se muestran que existieron dos piezas con baja calidad; obteniendo un nivel de calidad del 98,88\%.

Finalmente la ecuación (5) muestra el valor de OEE obtenido, este corresponde a un $95,55 \%$ y su interpretación se lleva a cabo mediante la evaluación propuesta en la teoría OEE, la cual sustenta que la etapa validada, al encontrarse por encima del $95 \%$, se traduce en un proceso de clase mundial.

$$
\begin{gathered}
\text { OEE }=(0,979) \times(0,978) \times(0,998)= \\
0,9555=95,55 \%
\end{gathered}
$$

Un valor OEE del $100 \%$ en la práctica es muy difícil de alcanzar, sin embargo es un parámetro referente para definir sistemáticamente procesos de mejora continua.

\section{CONCLUSIONES}

En este trabajo se presentó la técnica de modelado de sistemas productivos a través de RdP híbridas, con el objetivo de estudiar el comportamiento y apoyar en la toma de decisiones en el orden de mejorar estratégicamente el sistema mediante la integración del paradigma de producción esbelta.

Las RdP, por su naturaleza gráfica y formal, muestran ser una herramienta que permite una descripción fácilmente comprensible de un sistema, para modelar comportamientos que incluyen secuencias, concurrencia, paralelismo y toma de decisiones. Las alternativas de operación en sistemas de producción que pueden ser modeladas a través de ellas son bastante extensas y descriptivas. Un aspecto importante por resaltar es que las RdP gracias a sus propiedades nos permiten tomar en cuenta variables críticas, que a través de otro tipo de modelo podríamos olvidar considerar.

El impacto que tienen las redes de Petri en relación a un sistema productivo esbelto, se da en función de la capacidad que tienen las redes en modelar la complejidad de un sistema de estas características; teniendo la capacidad de representar elementos a través de lugares y eventos a través de transiciones. Las RdP permiten representar los diversos escenarios que se presentan en un sistema productivo; permiten identificar variables críticas, y conocer el comportamiento que conservará el sistema cuando los parámetros de una variable cambian.

Se ha validado una etapa de la RdP a través del indicador OEE y con ello se ha demostrado que la implementación correcta de métodos, herramientas y técnicas esbeltas generan sinergia en el sistema y en función de ello beneficios. La validación a través de tres parámetros fundamentales (disponibilidad, rendimiento y calidad) ayuda a calcular la efectividad productiva mediante un solo indicador y proporciona el detalle de las causas raíz de las pérdidas productivas, siendo éste el punto de partida de la mejora de productividad de la planta.

Es importante destacar que no todas las metodologías, técnicas y herramientas utilizadas en producción esbelta tendrán el mismo éxito al ser implantadas. Esto dependerá en gran medida de diferentes aspectos 
de tipo social, económico, cultural, político, factores como son el tipo de industria, y en forma muy particular en cómo se estudie, analice e implemente el paradigma.

\section{AGRADECIMIENTOS}

Los autores agradecen al Instituto Politécnico Nacional, CONACYT, COFAA IPN y al Departamento de Ciencias de Computación de la Universidad de Tecnología de Cottbus por las facilidades brindadas en el uso de software Snoopy Petri nets.

\section{REFERENCIAS}

[1] E. Jiménez y S. Hernández. "Marco Conceptual de la Cadena de Suministro: Un Nuevo Enfoque Logístico". SCT. México. 2002.

[2] J.P. van Gigch. "Teoría General de Sistemas". Trillas. México. 2006. ISBN: 9682475899.

[3] A. Morales, A. Morales y J. Rojas. "Alternativas de Modelos de Cadena de Suministro con Enfoque Sistémico”. CNIES. México. 2012.

[4] C. Chandra and J. Grabis. "Supply Chain Configuration: Concepts, Solutions, and Applications". Springer. New York, USA. 2007. ISBN: 9780387251905.

[5] L. von Bertalanffy. "Teoría General de los Sistemas". Fondo de Cultura Económico. México. 2006. ISBN: 9789681606275.

[6] J.R. Stock and D.M. Lambert. "Strategic Logistics Management". McGraw-Hill. Boston. 2001. ISBN: 9780975994900.

[7] K.E. Maani and R.Y. Cavana. "System Thinking, System Dynamics: Managing Change and Complexity". Pearson Education. New Zealand. 2007. ISBN: 9781877371035.

[8] W. Ríos. "Ingeniería de Sistemas". Serie Publicaciones Docentes. Universidad de Chile. Chile. 2001.

[9] SupplyChainCouncil. Supply Chain Operations Reference (SCOR®) Model: Overview. 8 de Abril de 2014. URL: http:// supply-chain.org

[10] D.M. Lambert. "Supply Chain Management: Processes, Partnerships, Performance". Supply Chain Management Institute. Florida. 2008. ISBN: 9780975994900.

[11] J.P. Womack, D.T. Jones and D. Ross. "The Machine That Changed the World: The Story of Lean Production". Free Press. New York, USA. 2007. ISBN: 9780743299794.
[12] Y. Juárez, J. Rojas, J. Medina y A. Pérez. "El Enfoque de Sistemas para la Aplicación de la Manufactura Esbelta". Científica ESIME. Vol. 15, pp. 35-42. 2011.

[13] L. Socconini. "Lean Manufacturing”. Norma. Mexico. 2008. ISBN: 9789700919324.

[14] A. Villaseñor y E. Galindo. "Manual de Lean Manufacturing". Limusa. México. 2009. ISBN: 9789681869755.

[15] J. Magalhaes. "Los 7 Desperdicios de la Manufactura Esbelta". Sistemas de Producción y Manufactura Esbelta. 2010.

[16] R. David and H. Alla. "Petri Nets for Modeling of Dynamic Systems: A Survey”. Automática. Vol. 30, pp. 175-202. 1994.

[17] M. Silva. "Las Redes de Petri en la Automática y la Informática". Editorial AC. Madrid. 2002. ISBN: 9788472880450.

[18] V. Padmavatimetta. "Modelling y Simulation of SNP Systems Using Petri Nets". PhD Computer Science and Engineering, Thapar University. Patiala, India. 2012.

[19] R. David and H. Alla. "Discrete, continuous and Hybrid Petri Nets". Heidelberg: Springer. Berlín, Alemania. 2010. DOI:10.1142/ S0218126698000079.

[20] A. Casanova and L. Cuatrecasas. "Lean Supply Chain Management". Profit. Barcelona, España. 2011. ISBN: 9788415330516.

[21] Y. Sugimori, K. Kusunoki, F. Cho and S. Uchikawa. "Toyota Production System and Kanban System Materialization of Just in Time and Respect for Human System". International Journal of Production Research. Vol. 15, pp. 553-564. 2007. DOI: 10.1080/00207547708943149.

[22] P. Thomas, A. Thomas and S. Belmokhtar. "Petri Net Control of a Kanban Loop". International Conference on Industrial Engineering and Systems Management. 2011.

[23] M. Heiner, M. Herajy, F. Liu, C. Rohr and M. Schwarick. "Snoopy: A Unifying Petri Net Tool". Applications and Theory of Petri Nets. Vol. 7347, pp. 398-407. 2012. DOI: $10.1007 / 978364231131422$.

[24] I. Flores, J. Figueras, A. Guasch, M.A. Mújica, M. Narciso y M.A. Piera. "Modelos de Simulación usando Simo y Redes de Petri". UNAM. Facultad de Ingeniería. México. 2013. ISBN: 9786070246890. 


\section{ANEXO 1}

Tabla 4. Lugares y Transiciones de la Red Total.

\begin{tabular}{|c|c|c|c|}
\hline Lugar & Función & Transición & Función \\
\hline p1 & Suministro & $\mathrm{t} 1$ & Transición Fuente \\
\hline p2 & Suministro & t2 & Transición Fuente \\
\hline p3 & Suministro & $\mathrm{t} 3$ & Transición Fuente \\
\hline $\mathrm{p} 4$ & Materia Prima: Caucho Natural & $\mathrm{t} 4$ & Inicio de Suministro de Materia Prima 1 \\
\hline $\mathrm{p} 5$ & Materia Prima: Caucho Sintético & t5 & Inicio de Suministro de Materia Prima 2 \\
\hline p6 & Materia Prima: Cordones de Nylon & t6 & Inicio de Suministro de Materia Prima 3 \\
\hline $\mathrm{p} 7$ & Corte del Fardo & $\mathrm{t} 7$ & Inicio de Operación p7 \\
\hline $\mathrm{p} 8$ & Laminado & t8 & $\begin{array}{l}\text { Fin de Operación p7 } \\
\text { Inicio de Operación p8 }\end{array}$ \\
\hline p9 & Materia Prima: Químicos Azufres & t9 & Transición Fuente \\
\hline $\mathrm{p} 10$ & Materia Prima: Carbón Negro & $\mathrm{t} 10$ & Transición Fuente \\
\hline p11 & Mezcla Intensiva & $\mathrm{t} 11$ & Inicio de Mezcla Intensiva \\
\hline p12 & Transporte a Estrujado de Alerón & $\mathrm{t} 12$ & $\begin{array}{l}\text { Fin de Mezcla Intensiva } \\
\text { Orden de Transporte a Línea de Ensamble Final }\end{array}$ \\
\hline p13 & Transporte a Tamizado & $\mathrm{t} 13$ & Inicio Operación p16 \\
\hline p14 & Transporte a Producción de Listones & $\mathrm{t} 14$ & $\begin{array}{l}\text { Fin de Operación p16 } \\
\text { Inicio de Operación p17 }\end{array}$ \\
\hline p15 & Transporte a Estrujado de Llanta & $\mathrm{t} 15$ & $\begin{array}{l}\text { Fin de Operación p17 } \\
\text { Transporte p18 }\end{array}$ \\
\hline $\mathrm{p} 16$ & Estrujado Alerón & $\mathrm{t} 16$ & Inicio Operación p19 \\
\hline p17 & Prensado & $\mathrm{t} 17$ & $\begin{array}{l}\text { Fin de Operación p19 } \\
\text { Inicio de Operación p20 }\end{array}$ \\
\hline p18 & Producto Terminado: Alerón & $\mathrm{t} 18$ & $\begin{array}{l}\text { Fin de Operación p20 } \\
\text { Inicio de Operación p21 }\end{array}$ \\
\hline p19 & Tamizado & $\mathrm{t} 19$ & $\begin{array}{l}\text { Fin de Operación p21 } \\
\text { Inicio de Operación p22 }\end{array}$ \\
\hline $\mathrm{p} 20$ & Laminado & $\mathrm{t} 20$ & $\begin{array}{l}\text { Fin de Operación p22 } \\
\text { Inicio de Operación p23 }\end{array}$ \\
\hline $\mathrm{p} 21$ & Estrujado & $\mathrm{t} 21$ & $\begin{array}{l}\text { Fin de Operación p23 } \\
\text { Inicio de Operación p24 }\end{array}$ \\
\hline $\mathrm{p} 22$ & Revestido & $\mathrm{t} 22$ & $\begin{array}{l}\text { Fin de Operación p24 } \\
\text { Orden de Transporte a Línea de Ensamble Final }\end{array}$ \\
\hline $\mathrm{p} 23$ & Montaje & $\mathrm{t} 23$ & Transición Fuente \\
\hline $\mathrm{p} 24$ & Prensado & $\mathrm{t} 24$ & Transición Fuente \\
\hline $\mathrm{p} 25$ & Producto Terminado: Cámaras & $\mathrm{t} 25$ & Inicio Operación p26 \\
\hline p26 & Producción de Listones & $\mathrm{t} 26$ & $\begin{array}{l}\text { Fin de Operación p26 } \\
\text { Inicio de Operación p29 }\end{array}$ \\
\hline p27 & Materia Prima: Alambre & $\mathrm{t} 27$ & $\begin{array}{l}\text { Fin de Operación p29 } \\
\text { Orden de Transporte a Línea de Ensamble de Llanta }\end{array}$ \\
\hline $\mathrm{p} 28$ & Materia Prima: Cordones & $\mathrm{t} 28$ & Inicio Operación p31 \\
\hline p29 & Envoltura & $\mathrm{t} 29$ & $\begin{array}{l}\text { Fin de Operación p31 } \\
\text { Inicio de Operación p32 }\end{array}$ \\
\hline $\mathrm{p} 30$ & Producto Terminado: Listones de Alambre & $\mathrm{t} 30$ & $\begin{array}{l}\text { Fin de Operación p32 } \\
\text { Orden de Transporte a Línea de Ensamble de Llanta }\end{array}$ \\
\hline p31 & Estrujado & $\mathrm{t} 31$ & Inicio Operación p33 \\
\hline p32 & Bandas de Llanta & $\mathrm{t} 32$ & $\begin{array}{l}\text { Fin de Operación p33 } \\
\text { Inicio de Operación p34 }\end{array}$ \\
\hline p33 & Calandrado & $\mathrm{t} 33$ & $\begin{array}{l}\text { Fin de Operación p34 } \\
\text { Inicio de Operación p35 }\end{array}$ \\
\hline p34 & Corte & $\mathrm{t} 34$ & $\begin{array}{l}\text { Fin de Operación p35 } \\
\text { Inicio de Operación p36 }\end{array}$ \\
\hline $\mathrm{p} 35$ & Bobinado & $\mathrm{t} 35$ & $\begin{array}{l}\text { Fin de Operación p36 } \\
\text { Orden de Transporte a Línea de Ensamble de Llanta } \\
\text { Inicio Operación p } 37\end{array}$ \\
\hline p36 & Cordones de Llanta & $\mathrm{t} 36$ & Inicio Operación p38 \\
\hline p37 & Construcción de la Llanta & $\mathrm{t} 37$ & $\begin{array}{l}\text { Fin de Operación p38 } \\
\text { Inicio de Operación p39 }\end{array}$ \\
\hline p38 & Proceso Llanta Verde & $\mathrm{t} 38$ & $\begin{array}{l}\text { Fin de Operación p39 } \\
\text { Inicio de Operación p40 }\end{array}$ \\
\hline p39 & Apertura & $\mathrm{t} 39$ & $\begin{array}{l}\text { Fin de Operación p } 40 \\
\text { Inicio de Operación p41 }\end{array}$ \\
\hline $\mathrm{p} 40$ & Esparcido de Polvo & $\mathrm{t} 40$ & $\begin{array}{l}\text { Fin de Operación p } 41 \\
\text { Inicio de Operación p42 }\end{array}$ \\
\hline $\mathrm{p} 41$ & Bladder & $\mathrm{t} 41$ & $\begin{array}{l}\text { Fin de Operación p42 } \\
\text { Inicio de Operación p43 }\end{array}$ \\
\hline
\end{tabular}




\begin{tabular}{|c|c|c|c|}
\hline Lugar & Función & Transición & Función \\
\hline $\mathrm{p} 42$ & Prensa de Curado & $\mathrm{t} 42$ & $\begin{array}{l}\text { Fin de Operación p43 } \\
\text { Inicio de Operación p44 }\end{array}$ \\
\hline $\mathrm{p} 43$ & Inflado & $\mathrm{t} 43$ & $\begin{array}{l}\text { Fin de Operación p44 } \\
\text { Inicio de Operación p45 }\end{array}$ \\
\hline $\mathrm{p} 44$ & Recorte & $\mathrm{t} 44$ & $\begin{array}{l}\text { Fin de Operación p45 } \\
\text { Inicio de Operación p46 }\end{array}$ \\
\hline $\mathrm{p} 45$ & Balanceo & $\mathrm{t} 45$ & $\begin{array}{l}\text { Fin de Operación p46 } \\
\text { Orden de Transporte a Línea de Ensamble Final }\end{array}$ \\
\hline $\mathrm{p} 46$ & Inspección & $\mathrm{t} 46$ & Ensamble de Componentes p18, p25 y p47 \\
\hline $\mathrm{p} 47$ & Producto Terminado: Llanta & $\mathrm{t} 47$ & Fin de Operación p48 \\
\hline $\mathrm{p} 48$ & Ensamble de los 3 Componentes Principales & $\mathrm{t} 48$ & Orden de Transporte a Almacén \\
\hline $\mathrm{p} 49$ & Transporte de Producto Terminado & $\mathrm{t} 49$ & Orden de Distribución \\
\hline $\mathrm{p} 50$ & Almacén & $\mathrm{t} 50$ & Obtención del Producto por un Cliente Final \\
\hline $\mathrm{p} 51$ & Distribución & $\mathrm{t} 51$ & $\begin{array}{l}\text { Inicio de Operación en MH1 } \\
\text { Condición del sensor } \pi 1\end{array}$ \\
\hline $\mathrm{p} 52$ & Cliente Final & $\mathrm{t} 52$ & $\begin{array}{l}\text { Fin de Operación en MH1 } \\
\text { Almacenaje de pieza en Alm } 1 .\end{array}$ \\
\hline $\mathrm{p} 53$ & Materia Prima & $\mathrm{t} 53$ & $\begin{array}{l}\text { Regreso del Manipulador a Posición Inicial } \\
\text { Avance a la Siguiente Etapa del Proceso } \\
\text { Detección de Inventario }\end{array}$ \\
\hline $\mathrm{p} 54$ & Proceso 1: Revestido & $\mathrm{t} 54$ & $\begin{array}{l}\text { Toma de pieza de Alm1 } \\
\text { Inicio de Operación en MH2 }\end{array}$ \\
\hline $\mathrm{p} 55$ & Almacén 1 & $\mathrm{t} 55$ & $\begin{array}{l}\text { Fin de Operación en MH2 } \\
\text { Almacenaje de pieza en Alm } 2\end{array}$ \\
\hline p56 & Proceso 2: Montaje & $\mathrm{t} 56$ & $\begin{array}{l}\text { Regreso del Manipulador a Posición Inicial } \\
\text { Avance a la Siguiente Etapa del Proceso } \\
\text { Detección de Inventario }\end{array}$ \\
\hline p57 & Almacén 2 & $\mathrm{t} 57$ & $\begin{array}{l}\text { Toma de pieza de Alm2 } \\
\text { Inicio de Operación en MH3 }\end{array}$ \\
\hline $\mathrm{p} 58$ & Proceso 3: Prensado & $\mathrm{t} 58$ & Fin de Operación en MH3 \\
\hline p59 & Producto terminado: Cámaras & t59 & $\begin{array}{l}\text { Condición del sensor } \pi 2 \\
\text { Avance a la Siguiente Etapa del Proceso }\end{array}$ \\
\hline p60 & Máquina 1 Disponible & t60 & $\begin{array}{l}\text { Transición Fuente: Generador de Fallas } \\
\text { Indicador de Falla en MH1 }\end{array}$ \\
\hline p61 & Espacio en Almacén 1 & t61 & $\begin{array}{l}\text { Transición Fuente: Generador de Fallas } \\
\text { Indicador de Falla en MH2 }\end{array}$ \\
\hline p62 & Máquina 2 Disponible & t62 & $\begin{array}{l}\text { Transición Fuente: Generador de Fallas } \\
\text { Indicador de Falla en MH3 }\end{array}$ \\
\hline p63 & Espacio en Almacén 2 & t63 & Inicio de Reparación en MH1 \\
\hline p64 & Máquina 3 Disponible & t64 & $\begin{array}{l}\text { Fin de Reparación en MH1 } \\
\text { Restablecimiento del Sistema en MH1 }\end{array}$ \\
\hline p65 & Robot Disponible & t65 & Inicio de Reparación en MH2 \\
\hline p66 & Procesamiento de Falla & t66 & $\begin{array}{l}\text { Fin de Reparación en MH2 } \\
\text { Restablecimiento del Sistema en MH2 }\end{array}$ \\
\hline p67 & Operación de Reparación de MH1 & t67 & Inicio de Reparación en MH3 \\
\hline p68 & Procesamiento de Falla & t68 & $\begin{array}{l}\text { Fin de Reparación en MH3 } \\
\text { Restablecimiento del Sistema en MH3 }\end{array}$ \\
\hline p69 & Operación de Reparación de MH2 & t69 & Señal de Estado de Producción \\
\hline $\mathrm{p} 70$ & Procesamiento de Falla & $\mathrm{t} 70$ & Transición Fuente: Generador de Fallas \\
\hline $\mathrm{p} 71$ & Operación de Reparación de MH3 & $\mathrm{t} 71$ & Transición Fuente: Generador de Fallas \\
\hline $\mathrm{p} 72$ & Inspección Operación 1 & $\mathrm{t} 72$ & Transición Fuente: Generador de Fallas \\
\hline $\mathrm{p} 73$ & Inspección Operación 2 & $\mathrm{t} 73$ & Inicio de Inspección Operación 1 \\
\hline $\mathrm{p} 74$ & Inspección Operación 3 & $\mathrm{t} 74$ & Inicio de Inspección Operación 2 \\
\hline $\mathrm{p} 75$ & Control Visual Falla 1 & $\mathrm{t} 75$ & Inicio de Inspección Operación 3 \\
\hline $\mathrm{p} 76$ & Control Visual Falla 2 & $\mathrm{t} 76$ & Orden de Retiro del Proceso \\
\hline p77 & Control Visual Falla 3 & $\mathrm{t} 77$ & $\begin{array}{l}\text { Orden de Kanban de Producción } \\
\text { Inicio de Operación de Manufactura }\end{array}$ \\
\hline p78 & Indicador de Flujo: Pieza en MH1 & $\mathrm{t} 78$ & $\begin{array}{l}\text { Fin de Operación de Manufactura } \\
\text { Orden de Movimiento }\end{array}$ \\
\hline p79 & Indicador de Flujo: Pieza en MH2 & $\mathrm{t} 79$ & Llegada a Almacén \\
\hline $\mathrm{p} 80$ & Indicador de Flujo: Pieza en MH3 & $\mathrm{t} 80$ & $\begin{array}{l}\text { Orden de Kanban de Producción } \\
\text { Inicio de Operación de Manufactura }\end{array}$ \\
\hline $\mathrm{p} 81$ & Indicador Visual de Producción & $\mathrm{t} 81$ & $\begin{array}{l}\text { Fin de Operación de Manufactura } \\
\text { Orden de Movimiento }\end{array}$ \\
\hline $\mathrm{p} 82$ & Reporte de Calidad en Operación 1 & $\mathrm{t} 82$ & Llegada a Almacén \\
\hline $\mathrm{p} 83$ & Reporte de Calidad en Operación 2 & $\mathrm{t} 83$ & $\begin{array}{l}\text { Orden de Kanban de Producción } \\
\text { Inicio de Operación de Manufactura }\end{array}$ \\
\hline
\end{tabular}




\begin{tabular}{|c|c|c|c|}
\hline Lugar & Función & Transición & Función \\
\hline p84 & Reporte de Calidad en Operación 3 & $\mathrm{t} 84$ & $\begin{array}{l}\text { Fin de Operación de Manufactura } \\
\text { Orden de Movimiento }\end{array}$ \\
\hline $\mathrm{p} 85$ & Depósito de Piezas Rechazadas & $\mathrm{t} 85$ & Llegada a Almacén \\
\hline p86 & Control Visual Mala Calidad 1 & t86 & $\begin{array}{l}\text { Orden de Kanban de Retiro } \\
\text { Inicio de Operación de Manufactura }\end{array}$ \\
\hline p87 & Control Visual Mala Calidad 2 & $\mathrm{t} 87$ & $\begin{array}{l}\text { Fin de Operación de Manufactura } \\
\text { Orden de Distribución }\end{array}$ \\
\hline $\mathrm{p} 88$ & Control Visual Mala Calidad 3 & $\mathrm{t} 88$ & Llegada de Producto a Cliente \\
\hline p89 & Almacén & t89 & Transición Fuente: Generador de Demanda \\
\hline p90 & Operación de Manufactura & t90 & Orden de Kanban de Retiro \\
\hline p91 & Máquina Disponible & t91 & \\
\hline p92 & Flujo de Material & p103 & Transporte Disponible \\
\hline p93 & Transporte Disponible & p104 & Inventario \\
\hline p94 & Inventario & p105 & Flujo de Material \\
\hline $\mathrm{p} 95$ & Operación de Manufactura & p106 & Transporte Disponible \\
\hline p96 & Máquina Disponible & $\mathrm{p} 107$ & Distribución \\
\hline p97 & Flujo de Material & p108 & Cliente \\
\hline p98 & Transporte Disponible & p109 & Registro de Demanda \\
\hline p99 & Inventario & p110 & Kanban de Producción \\
\hline p100 & Operación de Manufactura & p111 & Kanban de Producción \\
\hline p101 & Máquina Disponible & p112 & Kanban de Producción \\
\hline p102 & Flujo de Material & p113 & Kanban de Retiro \\
\hline
\end{tabular}

